\title{
A graviton propagator for inflation
}

\author{
Tomas Janssen*and Tomislav Prokopec ${ }^{\dagger}$ \\ Institute for Theoretical Physics, University of Utrecht
}

October 31, 2018

ITP-UU-07/37, SPIN-07/25

\begin{abstract}
We construct the scalar and graviton propagator in quasi de Sitter space up to first order in the slow roll parameter $\epsilon \equiv-\dot{H} / H^{2}$. After a rescaling, the propagators are similar to those in de Sitter space with an $\epsilon$ correction to the effective mass. The limit $\epsilon \rightarrow 0$ corresponds to the $E(3)$ vacuum that breaks de Sitter symmetry, but does not break spatial isotropy and homogeneity. The new propagators allow for a self-consistent, dynamical study of quantum back-reaction effects during inflation.
\end{abstract}

\section{Introduction}

While inflation -the accelerated expansion of the early universe- was originally introduced to solve the horizon, flatness and cosmic relic problem [1] 2], it was soon realized that inflationary space-times have the interesting property of particle creation [3] [4] [5. This can be seen from a simple application of the energy-time uncertainty relation [6] 7] that states that a virtual particle-anti particle pair with energy $E$ and momenta $\pm k$ can exist a time $\delta t$ given by

$$
\int_{t}^{t+\delta t} d t^{\prime} E\left(t^{\prime}, k\right) \lesssim 1
$$

While in flat space $\delta t$ is very small, it can grow large in curved space-time. In particular in the context of cosmological inflation, one finds that for massless modes with wavelengths larger then the hubble radius, $\delta t$ can become infinite. This means that during inflation massless, infrared particles are created out of the vacuum. Since photons and massless fermions couple conformally, their production rate is suppressed

\footnotetext{
${ }^{*}$ T.Janssen2@phys.uu.nl
}

†T.Prokopec@phys.uu.nl 
by the scale factor. The only non-conformally coupled, massless particles are massless minimally coupled scalars and gravitons.

Based on the discussion above one must conclude that quantum effects from massless minimally coupled scalars and gravitons during inflation can be very substantial. Indeed, these effects are exactly the source for scalar and tensor cosmological perturbations [3]. The imprint scalar perturbations leave on the cosmic microwave background has been verified by WMAP [8]. Tensor perturbations might be detected by the Planck satellite.

In general doing quantum field theory on a curved background is very involving, but, due to its high amount of symmetry, much more can be done in the context of de Sitter space (section 2). Since in de Sitter space the scale factor grows exponentially with time, it is an excellent paradigm for an inflationary space. The $D$-dimensional propagators in de Sitter space are known [9] [10] [11] and therefore it is possible to use dimensional regularization to calculate many kinds of interesting and possibly observable quantum effects during inflation. For example a massless minimally coupled scalar with a quartic self-interaction has been studied [10] 13] [14. The resulting model shows a violation of the weak energy condition on cosmological scales. A non-minimally coupled, massive scalar with quartic self-interaction has also been studied [15]. In this model the radiative corrections to slow roll inflation where calculated and found to be unobservable.

Also scalar electrodynamics has been studied extensively [6] [16] [17] [18] [19] [20]. The vacuum polarization has been studied and it has been shown that in such a model superhubble photons acquire a mass. The contribution to the zero-point energy of these photons sources cosmological magnetic fields. Most recently the theory has been formulated stochastically [21].

Another interesting line of research is the study of quantum back-reaction on the vacuum 22] 23] 24] 25] 26] 27] 28]. In a pioneering study [22] [23], it was shown that the two loop contribution to the graviton one-point function slows inflation. The resulting model, where inflation is driven by a large cosmological constant and ends naturally due to quantum effects is an alternative to scalar field driven inflation. Moreover it might prove to be important for a better understanding of the cosmological constant problem[29]. However this calculation was done in $3+1$ dimensions, using a (time dependent) ultraviolet momentum cutoff. Since it is not clear whether the observed effect is a relic of the regularization procedure, it is important that this calculation is redone using dimensional regularization [30].

There are however several drawbacks to the use of de Sitter space. First of all, while de Sitter space provides an excellent framework to study properties of inflation, it is never truly realized in nature. Indeed, since in de Sitter space the Hubble parameter is per definition globally constant, it follows that if the universe was once de Sitter it is always de Sitter. From the fact that the universe is not de Sitter today, it follows that it was never de Sitter. The deviation of the inflationary universe from de Sitter space is even measurable through the deviation of the spectral index $n_{s}$ from scale invariance [2] [8]. When these deviations are small enough, one could work 
in a space that is 'locally' de Sitter, however in that case there is no control on the error one is making.

The second problem with de Sitter space is that it is non-dynamical. While space-time is certainly curved, all dynamics are governed by the Hubble parameter and the Hubble parameter is constant. Strictly speaking it is therefore inconsistent to study how space-time changes due to back-reaction effects if the calculation of these effects explicitly assumes that the background is constant. To self-consistently consider back-reaction, one must allow the Hubble parameter to change with time. Although in 22] 23] a distinction is made between the constant Hubble parameter $H$ and the - dynamical- physical observable $H_{\text {eff }}$, it is still not fully consistent. The reason is that the propagators are calculated using the constant $H$, where they should be calculated with the dynamical, time dependent $H_{\text {eff }}$.

The final issue with de Sitter space is the fact that it is not possible to construct a propagator for a massless minimally coupled scalar or for the graviton that respects the de Sitter symmetry [31. Usually this problem is circumvented by letting these propagators break the de Sitter symmetry, but not spatial isotropy and homogeneity. The argument is that it is these symmetries one expects to be present in the early universe, not the full de Sitter group (because of the problem described above). However, if we are forced to abandon de Sitter symmetry anyway, why not do the complete calculation in a space with only spatial isotropy and homogeneity, but otherwise similar to de Sitter space.

Constructing the propagator in this space, known as quasi de Sitter space, is exactly the aim of this paper. Quasi de Sitter space is a generalization of de Sitter space, where $H$ depends only mildly on time (see section 2.1). While this time dependence breaks the full de Sitter symmetry, it preserves spatial homogeneity and isotropy. We therefore find that there is no problem to describe massless minimally coupled scalars or gravitons in quasi de Sitter space. Moreover, since quasi de Sitter space is both dynamical and a more realistic approximation in many inflationary models then de Sitter space, the other two problems mentioned above are also resolved.

In section 2 we overview some properties of de Sitter and quasi de Sitter space-times. In sections 3 and 4 we calculate the scalar and graviton propagators respectively. In section 4 we also calculate the ghost propagator associated with our gauge fixing. We conclude in section 5 .

\section{2 de Sitter and quasi de Sitter space}

4-dimensional de Sitter space is the hypersurface given by

$$
-X_{0}^{2}+X_{1}^{2}+X_{2}^{2}+X_{3}^{2}+X_{4}^{2}=\frac{1}{H^{2}}
$$

embedded in 5-dimensional Minkowski space-time, where $H$ is a constant parameter known as the Hubble parameter. de Sitter space is a solution to the Einstein 
equations with a positive cosmological constant, in which case

$$
H^{2}=\frac{\Lambda}{3} \text {. }
$$

The isometry group of de Sitter space, $S O(1,4)$, is manifest in this embedding. We will use flat coordinates, which cover only half of the de Sitter manifold, given by $(i=1,2,3)$

$$
\begin{aligned}
X_{0} & =\frac{1}{H} \sinh (H t)+\frac{H}{2} x_{i} x^{i} e^{H t}, \\
X_{i} & =e^{H t} x_{i}, \\
X_{4} & =\frac{1}{H} \cosh (H t)-\frac{H}{2} x_{i} x^{i} e^{H t}, \\
-\infty & <t, x_{i}<\infty .
\end{aligned}
$$

In these coordinates the metric reads

$$
g_{\mu \nu}=\operatorname{diag}\left(-1, a^{2}, a^{2}, a^{2}\right) \quad a=e^{H t},
$$

such that $\dot{a} / a=H$. The coordinates (6) can be written in conformal form by changing coordinates to conformal time $\eta$ defined as $a d \eta=d t$. The metric becomes:

$$
g_{\mu \nu}=a^{2} \eta_{\mu \nu}, \quad a=-\frac{1}{H \eta}, \quad \eta_{\mu \nu}=\operatorname{diag}(-1,1,1,1), \quad(\eta<0) .
$$

We define the de Sitter invariant distance functions

$$
Z(X ; \tilde{X})=H^{2} \eta_{A B} X^{A} \tilde{X}^{B}=1-\frac{1}{2} Y(X ; \tilde{X}) .
$$

In conformal coordinates these functions read

$$
\begin{aligned}
z_{++}(x ; \tilde{x}) & =1-\frac{1}{2} y_{++}(x ; \tilde{x}) \\
y_{++}(x ; \tilde{x}) & =\frac{\Delta x_{++}^{2}(x ; \tilde{x})}{\eta \tilde{\eta}} \\
\Delta x_{++}^{2}(x ; \tilde{x}) & =-(|\eta-\tilde{\eta}|-i \varepsilon)^{2}+\|\vec{x}-\overrightarrow{\tilde{x}}\|^{2},
\end{aligned}
$$

where $Y(X ; \tilde{X})=y(x ; \tilde{x}), Z(X ; \tilde{X})=z(x ; \tilde{x})$, are the functions given in (7). $a=a(\eta)$, $\tilde{a}=a(\tilde{\eta})$ and $\varepsilon>0$ refers to the Feynman (time ordered) pole prescription. In order to apply the Schwinger-Keldysh (see e.g. the appendix of [32]) formalism we also define

$$
\begin{aligned}
& \Delta x_{+-}^{2}(x ; \tilde{x})=-(\eta-\tilde{\eta}+i \varepsilon)^{2}+\|\vec{x}-\overrightarrow{\tilde{x}}\|^{2} \\
& \Delta x_{-+}^{2}(x ; \tilde{x})=-(\eta-\tilde{\eta}-i \varepsilon)^{2}+\|\vec{x}-\overrightarrow{\tilde{x}}\|^{2} \\
& \Delta x_{--}^{2}(x ; \tilde{x})=-(|\eta-\tilde{\eta}|+i \varepsilon)^{2}+\|\vec{x}-\overrightarrow{\tilde{x}}\|^{2}
\end{aligned}
$$


from which $y_{+-}, y_{-+}$and $y_{--}$follow immediately. For the rest of this paper we use

$$
y \equiv y_{++}, \quad \Delta x^{2} \equiv \Delta x_{++}^{2}
$$

unless stated otherwise. When $\varepsilon=0$, the function $y$ is related to the geodesic length $\ell=\ell(x ; \tilde{x})$ between points $x$ and $\tilde{x}$ as,

$$
y(x ; \tilde{x})=4 \sin ^{2}\left(\frac{1}{2} H \ell(x ; \tilde{x})\right) .
$$

The function $y$ has the following properties: if $y=0, \tilde{x}$ lies on the lightcone of $x$. For $y<0, \tilde{x}$ lies in the future or past of $x$ and for $y>0, x$ and $\tilde{x}$ are space-like separated. If $y=4, \tilde{x}$ lies on the light cone of the antipodal point 1 of $x$ and if $y>4, \tilde{x}$ is in the future or past of the antipodal point.

\subsection{Quasi de Sitter space}

Quasi de Sitter space is defined by the requirement that $H$ depends only mildly on time:

$$
H=H_{0}-H_{0}^{2} \epsilon t+\mathcal{O}\left(\epsilon^{2}\right), \quad \quad \epsilon \equiv-\frac{\dot{H}}{H^{2}} \ll 1, \quad \dot{\epsilon}=\mathcal{O}\left(\epsilon^{2}\right) .
$$

While such a Hubble parameter does not correspond to an exact solution of Einstein's equations, it is a good approximation in most inflationary models. For example in scalar field models, where inflation is achieved by a minimally coupled scalar field $\phi$, slowly rolling in a potential $V, \epsilon$ is just the slow roll parameter

$$
\epsilon=\frac{1}{2 M_{p}^{2}}\left(\frac{d(V(\phi)) / d \phi}{V(\phi)}\right)^{2}
$$

and (12) is correct up to order $\epsilon^{2}$ [2]. The metric of quasi de Sitter space can be written in conformal coordinates as

$$
g_{\mu \nu}=a^{2} \eta_{\mu \nu}
$$

where the scale factor is given by

$$
a=-\frac{1}{H_{0} \eta}(1+(1+\ln (a)) \epsilon)+\mathcal{O}\left(\epsilon^{2}\right)
$$

Since we know from (5) that $t=\ln (a) / H_{0}+\mathcal{O}(\epsilon)$, this is equal to

$$
a=-\frac{1}{\eta H}(1+\epsilon)+\mathcal{O}\left(\epsilon^{2}\right)
$$

If we take flat spacial slicings, the symmetry group of quasi de Sitter space is $E(3)$, which corresponds to spatial homogeneity and isotropy (invariance under translations and rotations).

\footnotetext{
${ }^{1}$ The antipodal point is obtained by the transformation of the coordinates (4) $X_{a} \rightarrow-X_{a}$, with $a=$ $(0 \ldots 4)$
} 


\section{The scalar propagator}

The Feynmann propagator

$$
\imath \Delta(x ; \tilde{x}) \equiv \imath \Delta(x ; \tilde{x})_{++}
$$

of a minimally coupled 2 scalar field with mass $m$ satisfies the following Klein-Gordon equation:

$$
\sqrt{-g}\left(\square-m^{2}\right) \imath \Delta(x ; \tilde{x})=\imath \delta^{D}(x-\tilde{x}),
$$

where $\square$ denotes the d'Alembertain

$$
\square \equiv \nabla^{\alpha} \nabla_{\alpha}
$$

and $\delta^{D}$ is the $D$-dimensional Dirac delta. In de Sitter space, one can solve for $\Delta(x ; \tilde{x})$ purely in terms of the de Sitter invariant distance function $y$ defined in (8) [9] [31. Replacing $y_{++}$with $y_{--}$gives the anti-time ordered propagator (which has the opposite sign in front of the delta function), while replacing it with $y_{+-}$and $y_{-+}$ gives the two Wightman propagators. The result, known as the Chernikov-Tagirov propagator [9], however becomes singular in the limit when the mass goes to zero. It was shown in [31] 33] that it is not possible to construct a physically meaningful de Sitter invariant propagator for the massless, minimally coupled scalar field. The reason is that in such a case the solution to (18) has an additional singularity when $\tilde{x}$ is on the lightcone of the antipodal point of $x$. Such a solution corresponds to the so called $\alpha$-vacuum. Although the issue is disputed, these vacua are usually considered unphysical 34] 35] 36, 37, 38.

In quasi de Sitter we find one can do something similar. It turns out that after a simple rescaling, we can construct a propagator purely in terms of the function $y$. Due to $\epsilon$ corrections, this propagator now has a consistent massless limit. The $y$ function we use is the one defined in (8). One could think however of different definitions for $y$. For example the most general definition appears to be through the geodesic length (11). However the geodesic length in quasi de Sitter turns out to be a rather complex object and not very helpful in solving (18). An alternative definition of $y$ is

$$
y_{\text {alt }}(x ; \tilde{x})=a \tilde{a} H \tilde{H} \Delta x^{2}(x ; \tilde{x})
$$

While this definition is equivalent to (8) in de Sitter space, it differs by a factor $(1+2 \epsilon)$ in quasi de Sitter. We use the definition (8), since in that case the singularities of the general solution to (18) are at $y=0$ and $y=4$ and thus resembles the de Sitter solution most.

Our rescaling of the propagator is

$$
\Delta(x ; \tilde{x}) \rightarrow(a \tilde{a})^{\epsilon w} \Xi(y),
$$

\footnotetext{
${ }^{2}$ Our results straightforwardly generalize to a non-minimally coupled scalar by replacing $m^{2} \rightarrow m^{2}+\xi R$, with $R$ the Ricci scalar and $\xi$ a a coupling parameter.
} 
where $w$ is a constant parameter. We shall determine $w$ by the requirement that $\Xi$ is a function of $y$ only (strictly speaking $\Xi$ is not a function, but a distribution). We rewrite the left hand side of (18) in terms of $y$-derivatives:

$$
\begin{aligned}
a^{D-2}(a \tilde{a})^{\epsilon w}[ & \left(-\left(\partial_{0} y\right)^{2}+\left(\partial_{i} y\right)^{2}\right)\left(\frac{d}{d y}\right)^{2} \\
& \left.+\left[\left(-\partial_{0}^{2} y+\partial_{i}^{2} y\right)-(D-2+2 \epsilon w)\left(\frac{a^{\prime}}{a}\right)\left(\partial_{0} y\right)\right] \frac{d}{d y}-a^{2} \mu^{2}\right] \imath \Xi(y)
\end{aligned}
$$

where

$$
\mu^{2} \equiv(D-1) \epsilon w H_{0}^{2}+m^{2}+\mathcal{O}\left(\epsilon^{2}\right)
$$

and we used

$$
\frac{a^{\prime}}{a^{2}}=H, \quad \frac{a^{\prime \prime}}{a^{3}}=\dot{H}+2 H^{2} .
$$

An important point of this calculation is that, even if the mass of the field is zero, the effective mass $\mu$ as it appears in (22) is nonzero. Therefore we find the propagator of a massless scalar field in quasi de Sitter space does not suffer from the presence of $\alpha$-vacua. Indeed this is expected, since in order to construct the propagator for such a field in de Sitter space, one exactly breaks the de Sitter symmetry to the symmetry of quasi de Sitter space. The several terms in (22) evaluate to

$$
\begin{aligned}
a^{-2}\left[-\left(\partial_{0} y\right)^{2}+\left(\partial_{i} y\right)^{2}\right]= & H^{2}(1-2 \epsilon) y(4-y)+\mathcal{O}\left(\epsilon^{2}\right) \\
a^{-2}\left[\left(-\partial_{0}^{2} y+\partial_{i}^{2} y\right)-(D-2+2 \epsilon w)\left(\frac{a^{\prime}}{a}\right)\left(\partial_{0} y\right)\right]= & -H^{2}(1-2 \epsilon)(D(y-2) \\
& +\epsilon(D-2+2 w) y)
\end{aligned}
$$

where we made use of (15) and (16). The $\delta$-function is only sourced by the singular term in $\Xi$ for $y \rightarrow 0$. Putting everything together we find for the nonsingular terms that

$$
\left[y(4-y)\left(\frac{d}{d y}\right)^{2}-(D(y-2)+(D-2+2 w) y \epsilon) \frac{d}{d y}-(1+2 \epsilon) \frac{\mu^{2}}{H^{2}}\right] \imath \Xi(y)=0 .
$$

In order for $\Xi$ to be a function of $y$ only, the left hand side of (26) needs to be a function of $y$ only. The term $\mu^{2} / H^{2}$ can in general never be written as a function of $y$. However if we assume that our field is light, such that

$$
m^{2}=\mathcal{O}(\epsilon)
$$

$\mu^{2} / H^{2}$ is, up to order $\epsilon^{2}$ corrections, proportional to $\mu^{2} / H_{0}^{2}$ and thus a constant. The 
resulting equation is a hypergeometric equation with a general solution

$$
\begin{aligned}
\imath \Xi(y)= & A_{2} F_{1}\left[\frac{D-1}{2}+\frac{D-2+2 w}{2} \epsilon+\nu_{D}, \frac{D-1}{2}+\frac{D-2+2 w}{2} \epsilon-\nu_{D} ; \frac{D}{2} ; \frac{y}{4}\right] \\
+ & B_{2} F_{1}\left[\frac{D-1}{2}+\frac{D-2+2 w}{2} \epsilon+\nu_{D},\right. \\
& \left.\frac{D-1}{2}+\frac{D-2+2 w}{2} \epsilon-\nu_{D} ; \frac{D}{2}+(D-2+2 w) \epsilon ; 1-\frac{y}{4}\right],
\end{aligned}
$$

where

$$
\nu_{D}^{2}=\left(\frac{D-1}{2}+\frac{D-2+2 w}{2} \epsilon\right)^{2}-(1+2 w) \frac{\mu^{2}}{H_{0}^{2}} .
$$

The constants $A$ and $B$ can be fixed by looking at the singular terms. First of all we require that the propagator has no singularity at $y=4$. Such a singularity would correspond to an additional delta-source at the antipodal point ( $\alpha$-vacuum) and is considered unphysical. This requirement fixes $A=0$. We fix $B$ by requiring that the singularity at $y=0$ sources the $\delta$-function correctly. The $\delta$ function is sourced by the most singular term, which is given by

$$
\imath \Xi(y)_{\text {sing }}=B\left(\frac{y}{4}\right)^{1-D / 2} \frac{\Gamma[D / 2+(D-2+2 w) \epsilon] \Gamma[D / 2-1]}{\Gamma\left[\frac{D-1}{2}+\frac{D-2+2 w}{2} \epsilon+\nu_{D}\right] \Gamma\left[\frac{D-1}{2}+\frac{D-2+2 w}{2} \epsilon-\nu_{D}\right]} .
$$

The delta function is sourced by letting $\sqrt{-g} \square$ act on this term. We find

$$
\begin{aligned}
& \imath \delta^{D}(x-\tilde{x})=(a \tilde{a})^{\epsilon w} a^{D-2} \partial^{2} \imath \Xi(y)_{\text {sing }} \\
& =\frac{(a)^{2 \epsilon w} \Gamma[D / 2+(D-2+2 w) \epsilon] \Gamma[D / 2-1]}{\Gamma\left[\frac{D-1}{2}+\frac{D-2+2 w}{2} \epsilon+\nu_{D}\right] \Gamma\left[\frac{D-1}{2}+\frac{D-2+2 w}{2} \epsilon-\nu_{D}\right]} B 2^{D-2}(-a \eta)^{D-2} \partial^{2}\left(\frac{1}{\Delta x^{D-2}}\right)
\end{aligned}
$$

where $\Delta x^{2}$ is defined in (8). Depending on the propagator we calculate, we use one of the following relations

$$
\begin{aligned}
& \partial^{2}\left(\frac{1}{\Delta x_{++}^{D-2}}\right)=\frac{4 \pi^{D / 2}}{\Gamma[D / 2-1]} \imath \delta^{D}(x-\tilde{x}) \\
& \partial^{2}\left(\frac{1}{\Delta x_{--}^{D-2}}\right)=-\frac{4 \pi^{D / 2}}{\Gamma[D / 2-1]} \imath \delta^{D}(x-\tilde{x}) \\
& \partial^{2}\left(\frac{1}{\Delta x_{-+}^{D-2}}\right)=\partial^{2}\left(\frac{1}{\Delta x_{+-}^{D-2}}\right)=0 .
\end{aligned}
$$

If one uses the appropriate form of (18) they all give the following result

$$
B=\frac{\Gamma\left[\frac{D-1}{2}+\frac{D-2+2 w}{2} \epsilon+\nu_{D}\right] \Gamma\left[\frac{D-1}{2}+\frac{D-2+2 w}{2} \epsilon-\nu_{D}\right]}{\Gamma[D / 2+(D-2+2 w) \epsilon]} \frac{H^{D-2}}{(4 \pi)^{D / 2}(1+\epsilon)^{D-2} a^{2 \epsilon w}} .
$$

We can now fix $w$ in order that $B$ is independent of $a$ and $\eta$. To do this we write

$$
\frac{H^{D-2}}{a^{2 w \epsilon}}=H_{0}^{D-2}(1-\epsilon(D-2+2 w) \ln (a))+\mathcal{O}\left(\epsilon^{2}\right)
$$


and therefore if we choose

$$
w=1-\frac{D}{2}
$$

$\Xi$ is truly only a function of $y$ up to order $\epsilon^{2}$ corrections. Remarkably, for this choice of $w$, equations (26) and (28) reduce exactly to their analogues in de Sitter space, with only the mass term modified. The final propagator is

$$
\begin{aligned}
\imath \Delta(x ; \tilde{x})= & \frac{(a \tilde{a})^{-\epsilon(D / 2-1)}}{(1+\epsilon)^{D-2}} \frac{H_{0}^{D-2}}{(4 \pi)^{D / 2}} \frac{\Gamma\left[\frac{D-1}{2}+\nu_{D}\right] \Gamma\left[\frac{D-1}{2}-\nu_{D}\right]}{\Gamma\left[\frac{D}{2}\right]} \\
& \times{ }_{2} F_{1}\left[\frac{D-1}{2}+\nu_{D}, \frac{D-1}{2}-\nu_{D} ; \frac{D}{2} ; 1-\frac{y}{4}\right],
\end{aligned}
$$

with

$$
\nu_{D}^{2}=\left(\frac{D-1}{2}\right)^{2}-\frac{m^{2}}{H_{0}^{2}}+\frac{\epsilon}{2}(D-1)(D-2), \quad m^{2}=\mathcal{O}(\epsilon) .
$$

The $\Delta_{--}, \Delta_{+-}$and $\Delta_{-+}$propagators can be obtained by replacing $y_{++}$in (36) by $y_{--}, y_{+-}$and $y_{-+}$, respectively. The reason is that the constant $B$, given in (33), is the same for all propagators.

\subsection{The de Sitter limit}

In the limit $\epsilon \rightarrow 0$, the leading order term for the massive scalar propagator is

$$
\begin{aligned}
\lim _{\epsilon \rightarrow 0} \imath \Delta(x ; \tilde{x})= & \frac{H_{0}^{D-2}}{(4 \pi)^{D / 2}} \frac{\Gamma\left[\frac{D-1}{2}+\nu_{D}\right] \Gamma\left[\frac{D-1}{2}-\nu_{D}\right]}{\Gamma\left[\frac{D}{2}\right]} \\
& \times{ }_{2} F_{1}\left[\frac{D-1}{2}+\nu_{D}, \frac{D-1}{2}-\nu_{D} ; \frac{D}{2} ; 1-\frac{y}{4}\right]+\mathcal{O}(\epsilon)
\end{aligned}
$$

with

$$
\lim _{\epsilon \rightarrow 0} \nu_{D}^{2}=\left(\frac{D-1}{2}\right)^{2}-\frac{m^{2}}{H_{0}^{2}} .
$$

This is - as expected - the Chernikov-Tagirov propagator [9]. For a massless scalar the story is slightly more complicated, since the term

$$
\Gamma\left[\frac{D-1}{2}-\nu_{D}\right]
$$


becomes singular. We split off this singular term by rewriting the hypergeometric function in (36) as a series:

$$
\begin{aligned}
\Delta(x, \tilde{x})= & \frac{(a \tilde{a})^{-\epsilon(D / 2-1)}}{(1+\epsilon)^{D-2}} \frac{H_{0}^{D-2}}{(4 \pi)^{D / 2}} \frac{\Gamma[D / 2] \Gamma[1-D / 2]}{\Gamma\left[1 / 2+\nu_{D}\right] \Gamma\left[1 / 2-\nu_{D}\right]} \\
& \times\left[\sum_{n=1}^{\infty} \frac{\Gamma\left[\frac{D-1}{2}+\nu_{D}+n\right] \Gamma\left[\frac{D-1}{2}-\nu_{D}+n\right]}{\Gamma[D / 2+n] \Gamma[n+1]}\left(\frac{y}{4}\right)^{n}\right. \\
& -\sum_{n=-1}^{\infty} \frac{\Gamma\left[\frac{3}{2}+\nu_{D}+n\right] \Gamma\left[\frac{3}{2}-\nu_{D}+n\right]}{\Gamma[3-D / 2+n] \Gamma[n+2]}\left(\frac{y}{4}\right)^{n-D / 2+2} \\
& \left.+\frac{\Gamma\left[\frac{D-1}{2}+\nu_{D}\right] \Gamma\left[\frac{D-1}{2}-\nu_{D}\right]}{\Gamma[D / 2]}\right]
\end{aligned}
$$

Now we can easily expand around $\epsilon=0$ and obtain

$$
\begin{aligned}
\imath \Delta(x ; \tilde{x})_{m=0}= & \frac{H_{0}^{D-2}}{(4 \pi)^{D / 2}}\left[\frac{-2 \Gamma[D-1]}{(D-2) \Gamma[D / 2]} \frac{1}{\epsilon}\right. \\
& +\frac{\Gamma[D-1]}{\Gamma[D / 2]}\left(\ln (a \tilde{a})+2-\gamma_{E}+\psi(D-1)+\pi \cot \left(\frac{D \pi}{2}\right)\right) \\
& +\sum_{n=1}^{\infty} \frac{\Gamma[n+D-1]}{n \Gamma[D / 2+n]}\left(\frac{y}{4}\right)^{n} \\
& \left.-\sum_{n=-1}^{\infty} \frac{\Gamma[n+D / 2+1]}{(n-D / 2+2) \Gamma[n+2]}\left(\frac{y}{4}\right)^{n-D / 2+2}\right]+\mathcal{O}(\epsilon),
\end{aligned}
$$

where $\gamma_{E}$ is the Euler-Mascheroni constant and $\psi[z] \equiv \frac{d}{d z} \ln (\Gamma[z])$ denotes the digamma function. Apart from the finite constants $2-\gamma_{E}+\psi(D-1)$ and the $1 / \epsilon$ term (which is also a constant in our approximation), (42) exactly corresponds to the massless scalar propagator in [10]. Since a constant corresponds to a solution of the homogeneous equation (scalar condensate), it can always be added. This can be seen from the fact that in the massless case the hypergeometric function multiplying ' $A$ ' in (28) is to leading order just 1 and thus we are not forced to put $A=0$. The propagator in [10] is calculated by requiring that the inevitable breaking of the de Sitter symmetry (to avoid $\alpha$-vacua) by a massless scalar, is such that it does not break spacial homogeneity and isotropy (this is known as the $E(3)$ vacuum). Since those symmetries are the symmetries of quasi de Sitter space, it is expected that the de Sitter limit of the quasi de Sitter propagator would correspond up to a constant to (42).

\section{The graviton propagator}

Various attempts have been made in the past to calculate the graviton propagator in de Sitter space. In many works [39] 40] 41] 42] this is done by adding a de Sitter 
invariant gauge fixing term. However, it turns out that these Green's functions give a divergent response to a point mass and moreover they do not solve the classical invariant equation of motion [43]. Based on this Tsamis and Woodard conclude that there must be some deep, still not completely understood physical principle that forbids the use of de Sitter invariant gauge fixing terms[11] [4] [45]. This problem might be related to the issues concerning the linearization instability in de Sitter space [11] [44] [47] 48] [49. The point is that the linearization instability implies a constraint on the (classical) field equations. This constraint has a nonzero response to sources on the whole de Sitter manifold. However, if one then adds a de Sitter invariant gauge fixing term and calculates Green's functions, one finds that these Green's functions only respond to sources in the past light cone. Since this does not cover the whole manifold, one has changed classical physics 3

This problem is circumvented by working only on half of the de Sitter manifold and by using a gauge fixing term that breaks the de Sitter symmetry [44. If one calculates the graviton propagator in this way, one finds that the solution can be written in terms of three scalar propagators, multiplying some tensor structure [30] 44]. Since one of these propagators corresponds to a massless minimally coupled scalar, the graviton propagator inevitably breaks the de Sitter symmetry.

In this section we calculate the graviton propagator in quasi de Sitter space. Although slightly more complicated, the result is similar as in de Sitter space. Two differences are that our gauge fixing term, the analogue of the one used in [30] 44], does not break the quasi de Sitter symmetry. Moreover, as we have shown above, a massless minimally coupled scalar poses no problems in quasi de Sitter space.

\subsection{The graviton action}

We start our calculation with the Einstein-Hilbert action with a cosmological constant $\Lambda$, coupled to a scalar field $\phi$.

$$
S_{\mathrm{EH}}=\frac{1}{\kappa} \int \sqrt{-\hat{g}}(\hat{R}-(D-2) \Lambda)+S_{M},
$$

where

$$
\begin{aligned}
\kappa & =16 \pi G_{N} \\
S_{M} & =\int \sqrt{-g}\left(-\frac{1}{2} \partial_{\alpha} \phi \partial_{\beta} \phi g^{\alpha \beta}-V(\phi)\right)
\end{aligned}
$$

and the hats indicate 'background+perturbation'. We have added the matter action $S_{M}$ for consistency, since without it, the quasi de Sitter metric is not a solution to Einstein's equations. Notice that the addition of matter induces a mixing between the graviton and the scalar field. Taking this mixing into account is crucial for the

\footnotetext{
${ }^{3}$ The problem seems to arise as soon as one imposes an average gauge condition, as when one adds a gauge fixing term. In Proca theory the de Sitter invariant propagator has been calculated using an exact gauge condition [46]. This propagator does give the correct response.
} 
calculation of scalar cosmological perturbations [50]. The four dimensional propagators, with mixing, are calculated in [51]52] in power law expansion. A study of the mixing between the gravitational and the matter section in $D$ dimensions will be done elsewhere [53. Quasi de Sitter is a good approximation if the scalar field $\phi$ is slowly rolling in the potential $V$ [2]. Using the geometric quantities from appendix A, the Friedmann and fluid equation corresponding to (43) are found to be

$$
\begin{aligned}
& H^{2}=\frac{1}{D-1} \Lambda+\frac{\kappa}{(D-1)(D-2)}\left(\frac{1}{2} \dot{\phi}^{2}+V(\phi)\right) \\
& \ddot{\phi}+(D-1) H \dot{\phi}=-\frac{d V(\phi)}{d \phi},
\end{aligned}
$$

where a dot denotes a time derivative. By choosing the appropriate potential, (45) can describe any time evolution of the scale factor. The action (43) therefore describes - as far as the geometry is concerned - a completely general FLRW universe. To calculate the graviton action we decompose our metric in a background $g$ plus a perturbation $h$ [54]:

$$
\begin{aligned}
& \hat{g}_{\mu \nu}=g_{\mu \nu}+h_{\mu \nu} \\
& \hat{g}^{\mu \nu}=g^{\mu \nu}+\delta g^{\mu \nu}
\end{aligned}
$$

If we require that $\hat{g}^{\mu \alpha} \hat{g}_{\mu \beta}=\delta_{\beta}^{\alpha}$ and we raise and lower indices on the perturbation with the background metric: $h^{\mu}{ }_{\alpha}=g^{\mu \nu} h_{\nu \alpha}$, we find that

$$
\delta g^{\mu \nu}=-h^{\mu \nu}+h^{\mu}{ }_{\alpha} h^{\alpha \nu}+\mathcal{O}\left(h^{3}\right) .
$$

We expand the action in powers of the perturbation around the background as

$$
S=S_{0}+S_{1}+\frac{1}{2} S_{2}+\ldots,
$$

where it should be noted that, since $\delta g^{\mu \nu}$ is quadratic in $h, S_{1}$ contains a part quadratic in $h$. For the first two terms we get

$$
\begin{aligned}
& S_{0}=\frac{1}{\kappa} \int \sqrt{-g}(R-(D-2) \Lambda)+S_{M} \\
& S_{1}=-\frac{1}{\kappa} \int \sqrt{-g}\left((E E)_{\mu \nu} h^{\mu \nu}-(E E)_{\mu \nu} h_{\alpha}^{\mu} h^{\alpha \nu}\right)
\end{aligned}
$$

where

$$
\begin{aligned}
T_{\mu \nu} & \equiv-\frac{2}{\sqrt{-g}} \frac{\delta S_{M}}{\delta g^{\mu \nu}}=\left(\partial_{\mu} \phi\right)\left(\partial_{\nu} \phi\right)-g_{\mu \nu}\left(\frac{1}{2}\left(\partial_{\alpha} \phi\right)\left(\partial_{\beta} \phi\right) g^{\alpha \beta}+V(\phi)\right) \\
(E E)_{\mu \nu} & \equiv R_{\mu \nu}-\frac{1}{2} g_{\mu \nu} R+\frac{D-2}{2} \Lambda g_{\mu \nu}-\frac{\kappa}{2} T_{\mu \nu} .
\end{aligned}
$$


If we drop terms cubic in $h, S_{2}$ is given by

$$
\begin{gathered}
S_{2}=-\frac{1}{\kappa} \int \sqrt{-g}\left[\frac{1}{2} h h^{\mu \nu}(E E)_{\mu \nu}+\tilde{h}^{\mu \nu}\left(\delta R_{\mu \nu}\right)-\frac{1}{2} R h_{\mu \nu} h^{\mu \nu}+\frac{1}{2} h h^{\mu \nu} R_{\mu \nu}\right. \\
\left.+\frac{D-2}{2} \Lambda h_{\mu \nu} h^{\mu \nu}-\frac{\kappa}{2} h^{\mu \nu}\left(\delta T_{\mu \nu}\right)\right],
\end{gathered}
$$

where we have defined the trace reversed graviton:

$$
\tilde{h}_{\mu \nu} \equiv h_{\mu \nu}-\frac{1}{2} g_{\mu \nu} h, \quad h \equiv g^{\mu \nu} h_{\mu \nu} .
$$

Using the following identities [55]

$$
\begin{gathered}
\delta R_{\alpha \beta}=\frac{1}{2} g^{\lambda \rho}\left(\nabla_{\lambda} \nabla_{\alpha} h_{\rho \beta}+\nabla_{\lambda} \nabla_{\beta} h_{\rho \alpha}-\nabla_{\lambda} \nabla_{\rho} h_{\alpha \beta}-\nabla_{\alpha} \nabla_{\beta} h_{\lambda \rho}\right) \\
\tilde{h}^{\alpha \beta} g^{\lambda \rho} \nabla_{\lambda} \nabla_{\alpha} h_{\rho \beta}=\tilde{h}^{\alpha \beta} g^{\lambda \rho} \nabla_{\alpha} \nabla_{\lambda} h_{\rho \beta}+R_{\sigma \alpha} h_{\beta}^{\sigma} \tilde{h}^{\alpha \beta}-R_{\beta \sigma \alpha \lambda} h^{\sigma \lambda} \tilde{h}^{\alpha \beta}
\end{gathered}
$$

and removing boundary terms, we find

$$
\begin{aligned}
\frac{S_{2}}{2}= & \frac{1}{\kappa} \int \sqrt{-g}\left[\frac{1}{4} \tilde{h}^{\mu \nu}\left(\square g_{\alpha \mu} g_{\beta \nu}+2 R_{\alpha \mu \beta \nu}\right) h^{\alpha \beta}+\frac{1}{2}\left(\nabla_{\mu} \tilde{h}^{\mu \alpha}\right)\left(\nabla^{\nu} \tilde{h}_{\nu \alpha}\right)\right. \\
& \left.+\frac{\kappa}{4} h^{\mu \nu}\left(\delta T_{\mu \nu}\right)-\frac{\kappa}{4} T_{\mu \nu} h^{\alpha \nu} h_{\alpha}{ }^{\mu}-\frac{1}{2}\left(h^{\alpha \nu} h_{\alpha}{ }^{\mu}+\frac{1}{2} h h^{\mu \nu}\right)(E E)_{\mu \nu}\right]
\end{aligned}
$$

where the d'Alembertian is given in (19). Adding the part quadratic in $h$ from $S_{1}$ the total quadratic part of the action becomes:

$$
\begin{aligned}
S^{(2)}=\frac{1}{\kappa} \int \sqrt{-g}[ & \frac{1}{4} \tilde{h}^{\mu \nu}\left(\square g_{\alpha \mu} g_{\beta \nu}+2 R_{\alpha \mu \beta \nu}\right) h^{\alpha \beta}+\frac{1}{2}\left(\nabla_{\mu} \tilde{h}^{\mu \alpha}\right)\left(\nabla^{\nu} \tilde{h}_{\nu \alpha}\right) \\
& \left.+\frac{\kappa}{4}\left(h^{\mu \nu}\left(\delta T_{\mu \nu}\right)-T_{\mu \nu} h^{\alpha \nu} h_{\alpha}{ }^{\mu}\right)+\frac{1}{2} \tilde{h}^{\rho \nu} h_{\rho}{ }^{\mu}(E E)_{\mu \nu}\right]
\end{aligned}
$$

In the following we drop the $(E E)_{\mu \nu}$ terms from (56), since they are zero on-shell. The contribution to the graviton action from the matter action can be found from (50) and

$$
\delta T_{\mu \nu}=-h_{\mu \nu}\left(\frac{1}{2}\left(\partial_{\alpha} \phi\right)\left(\partial_{\beta} \phi\right) g^{\alpha \beta}+V(\phi)\right)+\frac{1}{2} g_{\mu \nu}\left(\partial_{\alpha} \phi\right)\left(\partial_{\beta} \phi\right) h^{\alpha \beta}
$$

to give

$$
\frac{\kappa}{4}\left(h^{\mu \nu}\left(\delta T_{\mu \nu}\right)-T_{\mu \nu} h^{\alpha \nu} h_{\alpha}{ }^{\mu}\right)=\frac{\kappa}{8}\left(\phi^{\prime}\right)^{2}\left(h h^{00}-2 h^{0 \lambda} h_{\lambda}^{0}\right)
$$

From (45) we can derive that

$$
\kappa\left(\phi^{\prime}\right)^{2}=-2(D-2) \dot{H} a^{2}
$$


where a prime denotes a derivative with respect to conformal time. Using this, the graviton action becomes

$$
\begin{aligned}
S_{g}^{(2)}=\frac{1}{\kappa} \int \sqrt{-g}[ & \frac{1}{4} \tilde{h}^{\mu \nu}\left(\square g_{\alpha \mu} g_{\beta \nu}+2 R_{\alpha \mu \beta \nu}\right) h^{\alpha \beta}+\frac{1}{2}\left(\nabla_{\mu} \tilde{h}^{\mu \alpha}\right)\left(\nabla^{\nu} \tilde{h}_{\nu \alpha}\right) \\
& \left.+\frac{D-2}{4} \dot{H}\left(h h^{0}{ }_{0}-2 h^{\lambda 0} h_{\lambda 0}\right)\right] .
\end{aligned}
$$

In any FLRW metric we can choose coordinates where the metric is conformally flat,

$$
g_{\mu \nu}=a^{2} \eta_{\mu \nu}
$$

A lengthy calculation with several partial integrations and using the quantities given in Appendix A gives apart from boundary terms the following result [44]:

$$
\begin{aligned}
S^{(2)}=a^{D+4}[ & \frac{1}{4} \psi^{\mu \nu}\left(\partial^{\alpha} \partial_{\alpha}-(D-2)\left(\frac{a^{\prime}}{a^{3}}\right) \partial_{0}\right) \psi_{\mu \nu} \\
& -\frac{1}{8} \psi\left(\partial^{\alpha} \partial_{\alpha}-(D-2)\left(\frac{a^{\prime}}{a^{3}}\right) \partial_{0}+4\left(\frac{a^{\prime}}{a^{2}}\right)^{2}\right) \psi \\
& -\frac{D-2}{2}\left(\left(\frac{a^{\prime}}{a^{2}}\right)^{2}+\dot{H}\right) \psi^{0 \nu} \psi_{0 \nu}+\frac{D-2}{4}\left(2\left(\frac{a^{\prime}}{a^{3}}\right)^{2}-\frac{a^{\prime \prime}}{a^{2}}+\dot{H}\right) \psi_{0}^{0} \psi \\
& \left.+\frac{1}{2}\left(\nabla_{\mu} \tilde{\psi}^{\mu \alpha}\right)\left(\nabla_{\nu} \tilde{\psi}_{\alpha}^{\nu}\right)\right]
\end{aligned}
$$

where

$$
h_{\mu \nu}=\sqrt{\kappa} a^{2} \psi_{\mu \nu}, \quad \tilde{\psi}_{\mu \nu}=\psi_{\mu \nu}-\frac{1}{2} g_{\mu \nu} \psi, \quad \psi=g^{\mu \nu} \psi_{\mu \nu} .
$$

We follow [44] and add the following gauge fixing term:

$$
-\frac{1}{2} a^{D+4}\left(\nabla_{\mu} \tilde{\psi}^{\mu \alpha}\right)\left(\nabla_{\nu} \tilde{\psi}_{\alpha}^{\nu}\right) .
$$

While this gauge fixing term breaks the de Sitter symmetry, it does respect the quasi de Sitter symmetry. Removing another total derivative and, using (24), we obtain the general form of the gauge fixed graviton action in FLRW space-time

$$
S_{G F}^{(2)}=\int a^{D} \eta^{\alpha \mu} \eta^{\beta \nu} \psi_{\alpha \beta}\left(\square_{S}\left(\frac{1}{4} \delta_{\mu}^{\rho} \delta_{\nu}^{\sigma}-\frac{1}{8} g_{\mu \nu} g^{\rho \sigma}\right)-\frac{D-2}{2}\left(H^{2}+\dot{H}\right) \delta_{\mu}^{0} \delta_{\nu}^{\rho} \delta_{0}^{\sigma}\right) \psi_{\rho \sigma},
$$

where

$$
\square_{S}=a^{-D} \partial_{\alpha} a^{D-2} \eta^{\alpha \beta} \partial_{\beta}
$$

is the d'Alembertian as it acts on a scalar field. 


\subsubsection{The Ghost lagrangian}

The ghost Lagrangian corresponding to our gauge fixing (64) is given by

$$
\mathcal{L}_{\text {ghost }}=-a^{D} \bar{V}^{\mu} \delta F_{\mu}
$$

where $\bar{V}$ is the anti-ghost field and

$$
\begin{aligned}
F_{\mu} & =\nabla_{\alpha} \tilde{\psi}^{\alpha}{ }_{\nu} \\
& =\partial_{\alpha} \psi^{\alpha}{ }_{\nu}-\frac{1}{2} \partial_{\nu} \psi+\left(\frac{a^{\prime}}{a}\right)\left(D \psi^{0}{ }_{\nu}-\delta_{\nu}^{0} \psi\right) .
\end{aligned}
$$

To calculate $\delta F_{\mu}$ we consider an infinitesimal coordinate transformation (indicated by 2)

$$
y^{2 \mu}=y^{\mu}+\kappa V^{\mu} .
$$

Under this transformation the metric $\hat{g}$ (see (46)) transforms as

$$
\hat{g}_{\mu \nu}^{2}(y)=\hat{g}_{\mu \nu}(y)-\kappa\left(\hat{g}_{\alpha \nu}(y) \partial_{\mu} V^{\alpha}+\hat{g}_{\beta \mu}(y) \partial_{\nu} V^{\beta}+V^{\rho} \partial_{\rho} \hat{g}_{\mu \nu}(y)\right)+\mathcal{O}\left(V^{2}\right)
$$

and thus we find for the variation of the rescaled graviton field

$$
\begin{aligned}
\delta \psi_{\mu \nu}= & -a^{-2}\left(g_{\alpha \nu} \partial_{\mu} V^{\alpha}+g_{\alpha \mu} \partial_{\nu} V^{\alpha}+2\left(\frac{a^{\prime}}{a}\right) g_{\mu \nu} V^{0}\right) \\
& -\kappa\left(\psi_{\alpha \nu} \partial_{\mu} V^{\alpha}+\psi_{\alpha \mu} \partial_{\nu} V^{\alpha}+2\left(\frac{a^{\prime}}{a}\right) \psi_{\mu \nu} V^{0}+V^{\rho} \partial_{\rho} \psi_{\mu \nu}\right) .
\end{aligned}
$$

Since we let $V^{\mu}$ be our anti-commuting ghost field, the second line will be cubic in the fields, when combined with (67), while for the purpose of this paper we are only interested in quadratic contributions. So dropping the second line, we obtain

$$
\begin{aligned}
\delta F_{\mu}=a^{-2}( & -g_{\alpha \mu} \partial^{\nu} \partial_{\nu} V^{\alpha}+(D-2) g_{\alpha \mu}\left(\frac{a^{\prime}}{a^{3}}\right) \partial_{0} V^{\alpha}-(D-2)\left(\frac{a^{\prime \prime}}{a^{3}}\right) g_{0 \nu} V^{0} \\
& \left.+3(D-2)\left(\frac{a^{\prime}}{a^{2}}\right)^{2} g_{0 \nu} V^{0}\right) .
\end{aligned}
$$

Now using (24) and (66) we find from (67)

$$
\mathcal{L}_{\text {ghost }}=a^{D} \eta_{\alpha \beta} \bar{V}^{\beta}\left(\delta_{\mu}^{\alpha} \square_{S}-(D-2)\left(H^{2}-\dot{H}\right) \delta_{\mu}^{0} \delta_{0}^{\alpha}\right) V^{\mu} .
$$

\subsection{Calculating the propagator}

It is a well known fact that the propagator should be invariant under the interchange of both legs

$$
\imath \Delta(x ; \tilde{x})=\imath \Delta(\tilde{x} ; x) .
$$

Looking at (65) we therefore see that, since $\square_{S}$ does not commute with $g_{\mu \nu}$, it acts on the other leg as

$$
\square_{S}\left(\eta^{\alpha \mu} \eta^{\beta \nu} \psi_{\alpha \beta}\right)=\square_{S}\left(a^{4} \psi^{\mu \nu}\right)
$$


Thus from (65) we find that the gauge fixed kinetic operator is

$$
\mathcal{D}_{\mu \nu}{ }^{\rho \sigma}=a^{D}\left[\square_{S}\left(\frac{1}{2} \delta_{\mu}^{(\rho} \delta_{\nu}^{\sigma)}-\frac{1}{4} \eta_{\mu \nu} \eta^{\rho \sigma}\right)-(D-2)\left(H^{2}+\dot{H}\right) \delta_{(\mu}^{0} \delta_{\nu}^{(\rho} \delta_{0}^{\sigma)}\right],
$$

where, because of (75) indices are effectively raised and lowered with $\eta^{\alpha \beta}$. The Feynmann propagator is defined by

$$
\mathcal{D}_{\mu \nu}{ }^{\rho \sigma}\left[\imath_{\rho \sigma} \Delta^{\alpha \beta}\right](x ; \tilde{x})=\imath \delta_{\mu}^{(\alpha} \delta_{\nu}^{\beta)} \delta^{D}(x-\tilde{x}) .
$$

Also indices of the propagator should be raised and lowered with the Minkowski metric $\eta_{\mu \nu}$. We solve this equation by making the following ansatz,

$$
\begin{aligned}
\imath\left[{ }_{\rho \sigma} \Delta^{\alpha \beta}\right](x, \tilde{x})= & a(x, \tilde{x}) \delta_{\rho}^{(\alpha} \delta_{\sigma}^{\beta)}+b(x, \tilde{x}) \delta_{(\rho}^{0} \delta_{\sigma)}^{(\alpha} \delta_{0}^{\beta)}+c(x, \tilde{x}) \eta_{\rho \sigma} \eta^{\alpha \beta} \\
& +d(x, \tilde{x})\left(\delta_{\rho}^{0} \delta_{\sigma}^{0} \eta^{\alpha \beta}+\delta_{0}^{\alpha} \delta_{0}^{\beta} \eta_{\rho \sigma}\right)+e(x, \tilde{x}) \delta_{\rho}^{0} \delta_{\sigma}^{0} \delta_{0}^{\alpha} \delta_{0}^{\beta} .
\end{aligned}
$$

This is the most general form that $\left[{ }_{\rho \sigma} \Delta^{\alpha \beta}\right]$ can take, consistent with the symmetries [44]. We insert this ansatz in (77) and, combining terms that multiply the same tensor structure, we obtain 6 differential equations (in the following $a, b, c, d$ and $e$ are functions of $x$ and $\tilde{x}$ )

$$
\begin{array}{rcc}
\delta_{\mu}^{(\alpha} \delta_{\nu}^{\beta)} & : & \frac{1}{2} \square_{S}(a)=\imath a^{-D} \delta^{D}\left(x-x^{\prime}\right) \\
\delta_{(\mu}^{0} \delta_{\nu)}^{(\alpha} \delta_{0}^{\beta)} & : & \frac{1}{2} \square_{S}(b)-(D-2)\left(H^{2}+\dot{H}\right)\left(a+\frac{1}{2} b\right)=0 \\
\eta_{\mu \nu} \eta^{\alpha \beta} & : & \square_{S}\left(\frac{2-D}{4} c-\frac{1}{4} a+\frac{1}{4} d\right)=0 \\
\delta_{\mu}^{0} \eta_{\nu 0} \eta^{\alpha \beta} & : & \frac{1}{2} \square_{S}(d)+(D-2)\left(H^{2}+\dot{H}\right)(c-d)=0 \\
\eta_{\mu \nu} \delta_{0}^{\alpha} \eta^{\beta 0} & : & \square_{S}\left(\frac{2-D}{4} d+\frac{1}{4} b+\frac{1}{4} e\right)=0 \\
\delta_{\mu}^{0} \delta_{\nu}^{0} \delta_{0}^{\alpha} \delta_{0}^{\beta} & : & \frac{1}{2} \square_{S}(e)-(D-2) H^{2}\left(\frac{1}{2} b-d+e\right)=0 .
\end{array}
$$

To solve this system first of all note that (81) and (83) just mean that

$$
\begin{aligned}
& d=-(D-2) c-a \\
& e=-(D-2) d-b=(D-2)^{2} x+(D-2) a-b
\end{aligned}
$$

So the only independent functions are $a, b$ and $c$. We redefine them as

$$
\begin{aligned}
a & =2 \Delta_{0} \\
b & =4\left(\Delta_{1}-\Delta_{0}\right) \\
c & =\frac{2}{(D-3)(D-2)}\left(\Delta_{2}-(D-2) \Delta_{0}\right),
\end{aligned}
$$

where $\Delta_{0,1,2}$ are functions of $x$ and $\tilde{x}$. We find that the system (79. .84) is solved by the three functions $\Delta_{n}$ obeying [27]

$$
\left(\frac{\square_{S}}{H^{2}}-n(D-n-1)(1-\epsilon)\right) \Delta_{n}=\frac{\imath \delta^{D}\left(x-x^{\prime}\right)}{a^{D} H^{2}},
$$


where $\epsilon$ is defined in (12). Equation (87) is just the scalar propagator equation (18). So, just like in de Sitter space [44, we find that we can write the graviton propagator in any FLRW spacetime in terms of three scalar propagators, with different couplings to the background curvature. Inserting (86) in the ansatz (78), we obtain the graviton propagator in any FLRW background

$$
\begin{aligned}
{\left[{ }_{\rho \sigma} \Delta^{\alpha \beta}\right]=} & \left(2 \bar{\delta}_{\rho}^{(\alpha} \bar{\delta}_{\sigma}^{\beta)}-\frac{2}{D-3} \bar{\eta}_{\rho \sigma} \bar{\eta}^{\alpha \beta}\right) \Delta_{0}+4 \delta_{(\rho}^{0} \bar{\delta}_{\sigma)}^{(\alpha} \delta_{0}^{\beta)} \Delta_{1} \\
& +\left[\frac{2}{(D-2)(D-3)}\left(\eta_{\rho \sigma}+(D-2) \delta_{\sigma}^{0} \delta_{\rho}^{0}\right)\left(\eta^{\alpha \beta}+(D-2) \delta_{0}^{\beta} \delta_{0}^{\alpha}\right)\right] \Delta_{2},
\end{aligned}
$$

where

$$
\bar{\eta}_{\mu \nu}=\eta_{\mu \nu}+\delta_{\mu}^{0} \delta_{\nu}^{0}
$$

How to solve (87) in a general FLRW space is not known 4 , but from section 3 we know how to solve these type of equations in quasi de Sitter space. In that case the functions $\Delta_{n}$ are given by (36) with

$$
\nu_{(D, \mathrm{n})}^{2}=\frac{(D-2 n-1)^{2}}{4}+\epsilon\left(\frac{1}{2}(D-1)(D-2)-n(D-n-1)\right) .
$$

Notice that in calculating (90) we did not use (37), since the requirement $m^{2}=\mathcal{O}(\epsilon)$ is not met. However, there is in this case no problem in using (29) to calculate $\nu_{D}$, since for the propagators (88) $\mu^{2} / H^{2}$ is a constant. The graviton propagator (88) is the main result of this paper.

\subsubsection{Comparison to mode function analysis}

It is an interesting exercise to see how our results compare to the standard treatment of gravitons in terms of mode functions. For this we only consider the 'physical' graviton $\psi_{i j}$, defined by requiring that

$$
\psi_{0 \mu}=0, \quad \psi=0 .
$$

We see that in this case the action (65) leads to the following equation of motion

$$
\square_{S} \psi_{i j}=a^{-2}\left(\partial^{2}-(D-2) \frac{a^{\prime}}{a} \partial_{0}\right) \psi_{i j}=0
$$

We rescale $\psi$ to the conformally coupled field $\psi^{c}$ defined as

$$
\psi_{i j} \equiv a^{-(D-2) / 2} \psi_{i j}^{c}
$$

The equation of motion for $\psi^{c}$ is in Fourier space

$$
\left[-\partial_{0}^{2}-k^{2}+\frac{(D-2)(D-4)}{4}\left(\frac{a^{\prime}}{a}\right)^{2}+\frac{D-2}{2} \frac{a^{\prime \prime}}{a}\right] \psi_{i j}^{c}(k)=0 .
$$

\footnotetext{
${ }^{4}$ It is however not hard to solve them if the equation of state parameter $w \equiv p_{\phi} / \rho_{\phi}$ is a constant, because in those cases, even though $\epsilon$ does not have to be small, it is constant.
} 
Using (15) we find that

$$
\left(\frac{a^{\prime}}{a}\right)^{2}=\frac{1+2 \epsilon}{\eta^{2}}, \quad \frac{a^{\prime \prime}}{a}=\frac{2+3 \epsilon}{\eta^{2}},
$$

where $\eta$ is conformal time. The equation of motion reduces [50] [56] [57] to

$$
\left[\partial_{0}^{2}+k^{2}-\left(\frac{D(D-2)}{4}+\frac{(D-2)(D-1)}{2} \epsilon\right) \frac{1}{\eta^{2}}\right] \psi_{i j}^{c}(k)=0 .
$$

This equation can be solved and the result is

$$
\psi_{i j}^{c}(k)=c_{1} \sqrt{\eta} H_{\nu}^{(1)}(k \eta)+c_{2} \sqrt{\eta} H_{\nu}^{(2)}(k \eta),
$$

where $H^{(1,2)}$ are Hankel functions of the first and second kind respectively and $c_{1,2}$ are constants. The index $\nu$ is given by

$$
\nu^{2}=\frac{(D-1)^{2}}{4}+\epsilon \frac{(D-1)(D-2)}{2} .
$$

Comparing this result with (90), we see that $\nu$ is exactly equal to $\nu_{(D, 0)}$. The reason that we recover the $n=0$ index, can be seen from (88) by the fact that only $\Delta_{0}$ acts on the physical graviton $\psi_{i j}$. This indicates that our propagator correctly reproduces the standard graviton two-point correlations, obtained from the mode functions.

From this calculation, we can also find a relation between $\nu$ and $n_{g}$, the spectral index of graviton perturbations

$$
n_{g}=\frac{d \ln \mathcal{P}_{g}}{d \ln k}
$$

The graviton power-spectrum $\mathcal{P}_{g}$ is, apart from some normalization

$$
\mathcal{P}_{g} \propto k^{D-1}\left|\psi_{i j}^{c}(k)\right|^{2} .
$$

We can calculate the power-spectrum in the infrared by expanding (97) for small $k$. The result is

$$
\psi_{i j}^{c}(k) \propto \eta^{1 / 2}(\eta k)^{-\nu}+\mathcal{O}\left(k^{-\nu+2}\right)
$$

and therefore we find that

$$
n_{g}=(D-1)-2 \nu=-(D-2) \epsilon+\mathcal{O}\left(\epsilon^{2}\right),
$$

which in $\mathrm{D}=4$ indeed corresponds to the well known result that $n_{g}=-2 \epsilon[2][50]$.

\subsubsection{Ghost propagator}

By similar arguments as in the previous section, we raise and lower indices on the ghost field with $\eta^{\mu \nu}$. It follows from (73) that the kinetic operator is given by

$$
\mathcal{D}_{\mu}{ }^{\alpha}=a^{D}\left(\delta_{\mu}^{\alpha} \square_{S}-(D-2)\left(H^{2}-\dot{H}\right) \delta_{\mu}^{0} \delta_{0}^{\alpha}\right) .
$$


The propagator is defined by

$$
\mathcal{D}_{\mu}{ }^{\alpha} \imath\left[{ }_{\alpha} \Delta^{\rho}\right](x ; \tilde{x})=\imath \delta_{\mu}^{\rho} \delta^{D}(x-\tilde{x})
$$

and we find that

$$
\imath\left[{ }_{\alpha} \Delta^{\rho}\right](x ; \tilde{x})=\imath \bar{\delta}_{\alpha}^{\rho} \hat{\Delta}_{0}(x ; \tilde{x})+\imath \delta_{\alpha}^{0} \delta_{0}^{\rho} \hat{\Delta}_{1}(x ; \tilde{x}),
$$

where the $\hat{\Delta}_{n}$ propagators satisfy

$$
\left(\frac{\square_{S}}{H^{2}}-n(D-n-1)(1+\epsilon)\right) \hat{\Delta}_{n}=\frac{\imath \delta^{D}\left(x-x^{\prime}\right)}{a^{D} H^{2}} .
$$

Just as (87), the functions $\hat{\Delta}$ can be computed as in section 3 . The solution is given by (36) with

$$
\hat{\nu}_{(D, \mathrm{n})}^{2}=\frac{(D-2 n-1)^{2}}{4}+\epsilon\left(\frac{1}{2}(D-1)(D-2)-3 n(D-n-1)\right) .
$$

\section{Conclusion}

While de Sitter space is an excellent framework to do calculations of quantum effects during inflation, it has two drawbacks. The first is that it is never realized in nature and the second is that it is not dynamical in the sense that the Hubble parameter is constant. To solve these problems one needs to do calculations in quasi de Sitter space, where first order correction in the slow roll parameter $\epsilon \equiv-\dot{H} / H^{2}$ to the background geometry are taken into account. Not only is quasi de Sitter space a more realistic approximation of the inflationary universe, it is also a dynamical space. Indeed, since $\dot{H}$ is nonzero, the Hubble parameter is allowed to change. Especially if one wants to consider back-reaction effects or calculate quantum corrections to tensor cosmological perturbations, taking these dynamics into account is crucial for self-consistency of the calculation.

In this paper the propagator for the graviton in quasi de Sitter space has been constructed in arbitrary dimension. In order to achieve this, first the propagator for a scalar field has been obtained. After a simple rescaling by a factor $(a \tilde{a})^{(1-D / 2) \epsilon}$, the scalar propagator in quasi de Sitter space has an identical structure as the corresponding scalar propagator in de Sitter space. The only difference is an order $\epsilon$ correction to the effective mass. Due to this correction, the massless limit is regular, where it is singular in de Sitter space.

Just as in de Sitter space, it is found that the graviton propagator in any FLRW space can be written as the sum of three scalar propagators with different amounts of coupling to the background geometry multiplying three different tensorial structures. If the background is quasi de Sitter, we are able to explicitly construct these scalar propagators. The graviton propagator in quasi de Sitter space allows for self-consistent calculations of graviton back-reaction and for quantum studies of tensor cosmological perturbations. 


\section{Acknowledgements}

We would like to thank Richard Woodard for illuminating discussions and comments.

\section{A Geometric quantities}

In this appendix we summarize some of the geometric quantities used in the text.

The results in this appendix are valid in any FLRW spacetime. In this paper we work (mostly) with conformally flat space-times where the metric is given by

$$
g_{\mu \nu}=a^{2} \eta_{\mu \nu} \quad \eta_{\mu \nu}=\operatorname{diag}(-1,1,1,1)
$$

We define the covariant derivative as 55

$$
\nabla_{\mu} V^{\alpha} \beta=\partial_{\mu} V_{\beta}^{\alpha}+\Gamma_{\mu \lambda}^{\alpha} V_{\beta}^{\lambda}-\Gamma_{\mu \beta}^{\lambda} V_{\lambda}^{\alpha}
$$

where the connection coefficients are given by

$$
\Gamma_{\mu \nu}^{\alpha}=\frac{a^{\prime}}{a}\left(\delta_{\mu}^{\alpha} \delta_{\nu}^{0}+\delta_{\nu}^{\alpha} \delta_{\mu}^{0}+\delta_{0}^{\alpha} \eta_{\mu \nu}\right),
$$

and a prime indicates a dervative with respect to conformal time. The Riemann tensor is defined as

$$
R_{\mu \beta \nu}^{\alpha}=\partial_{\beta} \Gamma_{\mu \nu}^{\alpha}-\partial_{\nu} \Gamma_{\beta \mu}^{\alpha}+\Gamma_{\beta \lambda}^{\alpha} \Gamma_{\mu \nu}^{\lambda}-\Gamma_{\nu \lambda}^{\alpha} \Gamma_{\beta \mu}^{\lambda}
$$

Using (110) we thus find

$$
\begin{aligned}
R_{\mu \beta \nu}^{\alpha}= & \left(\frac{a^{\prime \prime}}{a}-2\left(\frac{a^{\prime}}{a}\right)^{2}\right)\left(\delta_{\nu}^{\alpha} \delta_{\mu}^{0} \delta_{\beta}^{0}-\delta_{0}^{\alpha} \delta_{\nu}^{0} \eta_{\mu \beta}-\delta_{\beta}^{\alpha} \delta_{\nu}^{0} \delta_{\mu}^{0}+\delta_{\beta}^{0} \delta_{0}^{\alpha} \eta_{\mu \nu}\right) \\
& -\left(\frac{a^{\prime}}{a}\right)^{2}\left(\delta_{\nu}^{\alpha} \eta_{\mu \beta}-\delta_{\beta}^{\alpha} \eta_{\mu \nu}\right) \\
R_{\mu \nu}= & R^{\alpha}{ }_{\mu \alpha \nu} \\
= & \left(\frac{a^{\prime \prime}}{a}-2\left(\frac{a^{\prime}}{a}\right)^{2}\right)\left(\eta_{\mu \nu}-(D-2) \delta_{\mu}^{0} \delta_{\nu}^{0}\right)+\left(\frac{a^{\prime}}{a}\right)^{2}(D-1) \eta_{\mu \nu} \\
R= & R^{\mu}{ }_{\mu} \\
= & \left(\frac{a^{\prime \prime}}{a}-2\left(\frac{a^{\prime}}{a}\right)^{2}\right) 2(D-1)+\left(\frac{a^{\prime}}{a}\right)^{2} D(D-1)
\end{aligned}
$$

\section{References}

[1] A. H. Guth, Phys. Rev. D 23 (1981) 347.

[2] A. R. Liddle, arXiv:astro-ph/9901124.

[3] V. F. Mukhanov and G. V. Chibisov, JETP Lett. 33 (1981) 532 [Pisma Zh. Eksp. Teor. Fiz. 33 (1981) 549]. 
[4] A. A. Starobinsky, JETP Lett. 30 (1979) 682 [Pisma Zh. Eksp. Teor. Fiz. 30 (1979) 719].

[5] N. D. Birrell and P. C. W. Davies, Cambridge, Uk: Univ. Pr. (1982) 340p

[6] T. Prokopec and R. P. Woodard, Am. J. Phys. 72 (2004) 60 [arXiv:astro-ph/0303358].

[7] R. P. Woodard, arXiv:astro-ph/0310757.

[8] D. N. Spergel et al. [WMAP Collaboration], arXiv:astro-ph/0603449.

[9] N. A. Chernikov and E. A. Tagirov, Annales Poincare Phys. Theor. A 9 (1968) 109.

[10] V. K. Onemli and R. P. Woodard, Class. Quant. Grav. 19 (2002) 4607 [arXiv:gr-qc/0204065].

[11] R. P. Woodard, arXiv:gr-qc/0408002.

[12] R. P. Woodard, Nucl. Phys. Proc. Suppl. 148 (2005) 108 [arXiv:astro-ph/0502556].

[13] V. K. Onemli and R. P. Woodard, Phys. Rev. D 70 (2004) 107301 [arXiv:gr-qc/0406098].

[14] T. Brunier, V. K. Onemli and R. P. Woodard, Class. Quant. Grav. 22 (2005) 59 [arXiv:gr-qc/0408080].

[15] A. Bilandzic and T. Prokopec, arXiv:0704.1905 [astro-ph].

[16] T. Prokopec, O. Tornkvist and R. P. Woodard, Phys. Rev. Lett. 89 (2002) 101301 [arXiv:astro-ph/0205331].

[17] T. Prokopec, O. Tornkvist and R. P. Woodard, Annals Phys. 303 (2003) 251 [arXiv:gr-qc/0205130].

[18] T. Prokopec and R. P. Woodard, Annals Phys. 312 (2004) 1 [arXiv:gr-qc/0310056].

[19] T. Prokopec, N. C. Tsamis and R. P. Woodard, Class. Quant. Grav. 24 (2007) 201 [arXiv:gr-qc/0607094].

[20] K. Dimopoulos, T. Prokopec, O. Tornkvist and A. C. Davis, Phys. Rev. D 65 (2002) 063505 [arXiv:astro-ph/0108093].

[21] T. Prokopec, N. C. Tsamis and R. P. Woodard, arXiv:0707.0847 [gr-qc].

[22] N. C. Tsamis and R. P. Woodard, Annals Phys. 253 (1997) 1 [arXiv:hep-ph/9602316].

[23] N. C. Tsamis and R. P. Woodard, Nucl. Phys. B 474 (1996) 235 [arXiv:hep-ph/9602315].

[24] L. R. W. Abramo, R. H. Brandenberger and V. F. M. Mukhanov, arXiv:gr-qc/9702004. 
[25] L. R. W. Abramo, R. H. Brandenberger and V. F. Mukhanov, Phys. Rev. D 56 (1997) 3248 [arXiv:gr-qc/9704037].

[26] B. Losic and W. G. Unruh, Phys. Rev. D 74 (2006) 023511 [arXiv:gr-qc/0604122].

[27] T. Janssen and T. Prokopec, JCAP 0705 (2007) 010 [arXiv:gr-qc/0703050].

[28] L. R. Abramo and R. P. Woodard, Phys. Rev. D 65 (2002) 063516 [arXiv:astro-ph/0109273].

[29] N. C. Tsamis and R. P. Woodard, Phys. Lett. B 301 (1993) 351.

[30] N. C. Tsamis and R. P. Woodard, Annals Phys. 321 (2006) 875 [arXiv:gr-qc/0506056].

[31] B. Allen, Phys. Rev. D 32 (1985) 3136.

[32] S. Weinberg, Phys. Rev. D 72 (2005) 043514 [arXiv:hep-th/0506236].

[33] B. Allen and A. Folacci, Phys. Rev. D 35 (1987) 3771.

[34] M. B. Einhorn and F. Larsen, Phys. Rev. D 67 (2003) 024001 [arXiv:hep-th/0209159].

[35] N. Kaloper, M. Kleban, A. Lawrence, S. Shenker and L. Susskind, JHEP 0211 (2002) 037 [arXiv:hep-th/0209231].

[36] T. Banks and L. Mannelli, Phys. Rev. D 67 (2003) 065009 [arXiv:hep-th/0209113].

[37] U. H. Danielsson, JHEP 0212 (2002) 025 [arXiv:hep-th/0210058].

[38] K. Goldstein and D. A. Lowe, Nucl. Phys. B 669 (2003) 325 [arXiv:hep-th/0302050].

[39] E. G. Floratos, J. Iliopoulos and T. N. Tomaras, Phys. Lett. B 197 (1987) 373.

[40] B. Allen, Phys. Rev. D 34 (1986) 3670.

[41] B. Allen and M. Turyn, Nucl. Phys. B 292 (1987) 813.

[42] A. Higuchi and S. S. Kouris, Class. Quant. Grav. 18 (2001) 4317 [arXiv:gr-qc/0107036].

[43] I. Antoniadis and E. Mottola, J. Math. Phys. 32 (1991) 1037.

[44] N. C. Tsamis and R. P. Woodard, Commun. Math. Phys. 162 (1994) 217.

[45] S. P. Miao and R. P. Woodard, Class. Quant. Grav. 23 (2006) 1721 [arXiv:gr-qc/0511140].

[46] N. C. Tsamis and R. P. Woodard, arXiv:gr-qc/0608069.

[47] R. Penrose, Relativity, Groups and Topology: Lectures. Edited by C. DeWitt and B. DeWitt. Gordon and Breach, New York, 1964. pp. 565-584

[48] J. Bicak and P. Krtous, Phys. Rev. D 64 (2001) 124020 [arXiv:gr-qc/0107078]. 
[49] J. Bicak and P. Krtous, J. Math. Phys. 46 (2005) 102504 [arXiv:gr-qc/0602009].

[50] V. Mukhanov, "Physical foundations of cosmology," Cambridge, UK: Univ. Pr. (2005) $421 p$

[51] J. Iliopoulos, T. N. Tomaras, N. C. Tsamis and R. P. Woodard, Nucl. Phys. B 534 (1998) 419 [arXiv:gr-qc/9801028].

[52] L. R. Abramo and R. P. Woodard, Phys. Rev. D 65 (2002) 063515 [arXiv:astro-ph/0109272].

[53] T. Prokopec, G. I. Rigopoulos and T. Janssen In progress

[54] S. M. Christensen and M. J. Duff, Nucl. Phys. B 170 (1980) 480.

[55] S. M. Carroll, arXiv:gr-qc/9712019.

[56] V. F. Mukhanov, H. A. Feldman and R. H. Brandenberger, Phys. Rept. 215 (1992) 203.

[57] L. P. Grishchuk, Sov. Phys. JETP 40 (1975) 409 [Zh. Eksp. Teor. Fiz. 67 (1974) 825]. 